\title{
Life expectancy and cause of death in males and females with Fabry disease: Findings from the Fabry Registry
}

\author{
Stephen Waldek, FRCP $P^{1}$, Manesh R. Patel, MD', Maryam Banikazemi, MD ${ }^{3,4}$, Roberta Lemay, MPH ${ }^{5}$, \\ and Philip Lee, FRCP
}

\begin{abstract}
Purpose: To evaluate life expectancy and cause of death among patients with Fabry disease, an X-linked lysosomal storage disorder. Methods: Data from 2848 patients in the Fabry Registry were summarized using descriptive statistics. Life expectancy at birth was compared with that of the United States general population. Results: As of August 2008, 75 of 1422 males and 12 of 1426 females in the Fabry Registry were reported to have died. The 87 deceased patients were diagnosed at a much older age than other patients in the Fabry Registry: median age at diagnosis was 40 vs. 24 years in males and 55 vs. 33 years in females. The life expectancy of males with Fabry disease was 58.2 years, compared with 74.7 years in the general population of the United States. The life expectancy of females with Fabry disease was 75.4 years, compared with 80.0 years in the United States general population. The most common cause of death among both genders was cardiovascular disease. Most (57\%) patients who died of cardiovascular disease had previously received renal replacement therapy. Conclusions: Most deceased Fabry Registry patients exhibited serious cardiac and renal dysfunction. Late diagnosis may have contributed to the early deaths of these patients. Genet Med 2009:11(11):790-796.
\end{abstract}

Key Words: $\alpha$-galactosidase A, Anderson-Fabry disease, lysosomal storage disease, longevity, cardiovascular

F abry disease is a rare, X-linked metabolic disorder caused by insufficient activity of the lysosomal enzyme $\alpha$-galactosidase A. This leads to the accumulation of glycolipids with terminal $\alpha$-galactosidic linkages, particularly globotriaosylceramide (GL-3), in various tissues and cell types. ${ }^{1}$ The progressive accumulation of glycolipids eventually impairs organ function. The incidence of Fabry disease has been estimated as 1 in 40,000 to 1 in 117,000 live births. ${ }^{1,2}$ This may be an underestimate, as patients with milder forms of the disease are not always diagnosed. There is no known predilection of Fabry disease for any specific race or ethnicity.

From the ${ }^{1}$ Salford Royal NHS Foundation Trust, Manchester, United Kingdom; ${ }^{2}$ Division of Cardiovascular Medicine, Duke University School of Medicine, Durham, North Carolina; Departments of ${ }^{3}$ Neurology and ${ }^{4}$ Pediatrics, Columbia University College of Physicians and Surgeons, New York, New York; ${ }^{5}$ Biomedical Data Sciences and Informatics, Genzyme Corporation, Cambridge, Massachusetts; and ${ }^{6} \mathrm{Charles}$ Dent Metabolic Unit, National Hospital for Neurology and Neurosurgery, Queen Square, London, United Kingdom.

Stephen Waldek, FRCP, Salford Royal NHS Foundation Trust, Manchester M6 8HD, United Kingdom. E-mail: steve.waldek@srht.nhs.uk.

Disclosure: The authors declare no conflict of interest

Submitted for publication April 29, 2009.

Accepted for publication July 8, 2009

Published online ahead of print September 10, 2009.

DOI: 10.1097/GIM.0b013e3181bb05bb
The early symptoms of Fabry disease, including neuropathic pain in the extremities, hypohidrosis, and gastrointestinal symptoms such as abdominal pain, diarrhea, and food intolerance, typically begin during childhood. ${ }^{3-5}$ Later in life, many patients develop life-threatening disease manifestations, including chronic renal disease, cardiovascular disease, and strokes. ${ }^{6-11}$ The signs and symptoms of Fabry disease are generally most severe among hemizygous males; however, heterozygous females often exhibit serious clinical manifestations affecting quality of life and longevity. ${ }^{7,10,12,13}$ Enzyme replacement therapy (ERT) with recombinant human $\alpha$-galactosidase A reduces plasma GL-3 levels and accumulation of GL-3 in tissue, attenuating many manifestations of Fabry disease, ${ }^{14-16}$ including stabilization, of estimated glomerular filtration rate over time $^{18,19}$ and reduction of cardiomyopathy. ${ }^{20,21}$

Lifespan is considerably shortened in males with Fabry disease. Before renal dialysis and renal transplants became widely available, the average age at death among males with Fabry disease was reported as $41-42$ years. $^{22,23}$ The increased availability of renal replacement therapy over the past several decades has extended the lifespan of Fabry patients. However, life expectancy of patients with Fabry disease has not been formally analyzed. Since 2001, five studies of relatively small cohorts of patients with Fabry disease have reported survival data ${ }^{6-8,13,24}$ and one study has reported cause of death data. ${ }^{24}$ In these studies, males were reported to survive to a mean or median age of 50-57 years $6,8,13,24$ and females to a mean or median age of 64-72 years. ${ }^{7,13,24}$ The Fabry Registry, which tracks the natural history and outcomes of patients with Fabry disease, had enrolled 2848 patients, including 1422 males and 1426 females, as of August 2, 2008. Detailed analyses of life expectancy, clinical events, and cause of death among males and females in this much larger cohort will provide valuable current information about longevity and mortality of patients with Fabry disease.

\section{MATERIALS AND METHODS}

The Fabry Registry is an ongoing, observational database that compiles clinical and investigative data on patients with Fabry disease. It began enrolling patients and collecting data in April 2001. All patients with Fabry disease are eligible for enrollment, regardless of age, gender, symptoms, or whether they are receiving ERT. Patient and physician participation is voluntary. All patients provided informed consent through local institutional review boards/ethics committees and may decline to participate or withdraw consent at any time.

Data collected by the Fabry Registry are entered into a database through a set of standardized case report forms. Data were analyzed using SAS statistical software version 8 (SAS Institute Inc., Cary, NC) and summarized using descriptive statistics. All Fabry Registry patients with assessment data were included in these analyses, regardless of enzyme treatment 
status. The cause of death was reported by treating physicians. Patients who were reported as deceased but for whom the date of death was unavailable were excluded from the analyses. The number of person-years of follow-up was calculated from the date of birth until the date of death or, for living patients, from the date of birth until the date of each patient's last assessment. Life expectancy at birth was computed according to standard life table methods ${ }^{25}$ and compared with the United States general population. ${ }^{26}$ Patients' ethnicities were self-reported and are included as a standard part of demographic characterization of the cohort.

Significant clinical events were categorized as cardiovascular, cerebrovascular, or renal. Cardiovascular events were defined as myocardial infarction, significant cardiac procedures (e.g., pacemaker placement, coronary bypass, stent placement, valve replacement), arrhythmia, angina pectoris, or congestive heart failure. Cerebrovascular events were defined as strokes. Renal events were defined as chronic dialysis ( $\geq 40$ days) or renal transplantation.

\section{RESULTS}

As of August 2, 2008, 75 of 1422 males and 12 of 1426 females in the Fabry Registry had died. Demographic data are summarized in Table 1. No major differences in ethnicity or in geographic region were observed between deceased patients and living patients. As shown in Table 1, the median age at death was 54.3 years in males (range, $31.2-84.8$ ) and 62.0 years in females (range, 39.9-76.1). Both living and deceased males reported the onset of Fabry symptoms at a median age of 10 years. The median age at symptom onset was younger in living females (15.2 years) than in deceased females (22.1 years), but this difference may not be meaningful, given that there were only 12 deceased females in the Fabry Registry (Table 1). Deceased patients were diagnosed with Fabry disease at much older ages than living patients. Deceased males were diagnosed at a median age of 39.8 years (range, $7.8-81.0$ ), whereas living males were diagnosed at a median age of 24.4 years (range,

Table 1 Demographic characteristics of deceased and living Fabry Registry patients

\begin{tabular}{|c|c|c|c|c|}
\hline \multirow[b]{2}{*}{ Parameter } & \multicolumn{2}{|c|}{ Males } & \multicolumn{2}{|c|}{ Females } \\
\hline & Deceased $(N=75)$ & Alive $(N=1347)$ & Deceased $(N=12)$ & Alive $(N=1414)$ \\
\hline \multicolumn{5}{|l|}{ Ethnicity, $n(\%)$} \\
\hline White & $66(88.0)$ & $1007(74.8)$ & $10(83.3)$ & $1125(79.6)$ \\
\hline Black & 0 & $28(2.1)$ & 0 & $23(1.63)$ \\
\hline Hispanic & $1(1.3)$ & $106(7.9)$ & $1(8.3)$ & $90(6.4)$ \\
\hline Asian & $2(2.7)$ & $54(4.0)$ & 0 & $18(1.3)$ \\
\hline Other & $3(4.0)$ & $48(3.6)$ & 0 & $33(2.3)$ \\
\hline Not specified & $3(4.0)$ & $104(7.7)$ & $1(8.3)$ & $125(8.8)$ \\
\hline \multicolumn{5}{|c|}{ Geographic region, $n(\%)$} \\
\hline United States & $29(38.7)$ & $486(36.1)$ & $6(50.0)$ & $483(34.2)$ \\
\hline Europe & $36(52.0)$ & $583(43.3)$ & $4(33.3)$ & $673(47.6)$ \\
\hline Rest of the world & $7(9.3)$ & $278(28.6)$ & $2(16.7)$ & $258(18.3)$ \\
\hline \multicolumn{5}{|c|}{ Age at death or last follow-up (yr) } \\
\hline Mean (SD) & $53.7(9.82)$ & $36.2(15.17)$ & $60.9(12.76)$ & $40.7(17.47)$ \\
\hline Median (range) & $54.3(31.2-84.8)$ & $37.6(1.4-82.3)$ & $62.0(39.9-76.1)$ & $41.9(0.2-84.1)$ \\
\hline \multicolumn{5}{|c|}{ Age at first Fabry symptom (yr) } \\
\hline$N$ & 61 & 1055 & 9 & 811 \\
\hline Mean (SD) & $17.3(16.12)$ & $14.1(12.39)$ & $22.3(13.86)$ & $21.6(16.50)$ \\
\hline Median (range) & $10.2(4.2-68.0)$ & $9.7(0-73.4)$ & $22.1(6.7-43.4)$ & $15.2(0-77.7)$ \\
\hline \multicolumn{5}{|c|}{ Age at Fabry diagnosis (yr) } \\
\hline$N$ & 74 & 1327 & 12 & 1349 \\
\hline Mean (SD) & $40.6(15.52)$ & $26.5(15.72)$ & $53.3(15.62)$ & $33.3(17.83)$ \\
\hline Median (range) & $39.8(7.8-81.0)$ & $24.4(0-79.8)$ & $55.4(23.8-72.5)$ & $32.7(0-80.6)$ \\
\hline \multicolumn{5}{|l|}{ Year of diagnosis } \\
\hline$N$ & 74 & 1327 & 12 & 1349 \\
\hline Mean (SD) & $1991(11.5)$ & $1996(10.0)$ & $1997(5.9)$ & $1999(9.2)$ \\
\hline Median (range) & 1992 (1966-2007) & $2001(1953-2008)$ & 1999 (1987-2005) & $2003(1950-2008)$ \\
\hline
\end{tabular}


0-79.8). Similarly, deceased females were diagnosed at a median age of 55.4 years (range, 23.8-72.5), compared with 32.7 years for living females (range, 0-80.6). In chronological terms, living patients were diagnosed more recently than deceased patients. Deceased males and females were diagnosed in median year 1992 and 1999, respectively, compared with 2001 and 2003 for living Fabry Registry males and females (Table 1).

As shown in Figure 1, most males died between the ages of 40 and 60 years, whereas most females died at ages older than 60 years. No Fabry Registry patients died before the age of 30 years. Sixty-one of the 75 deceased males $(81.3 \%)$ and 5 of the 12 deceased females $(41.7 \%)$ were known to have received ERT, which became commercially available in Europe 2001 and in the United States in 2003. The median length of time that these patients were on ERT was 12 months in males and 4 months in females. The risk of mortality among all patients in the Fabry Registry is shown in Figure 2. In males, the mortality rate increased from age 40 years onward and increased more rapidly at each 5-year increment over the age of 50 years. As expected, male patients were documented to have a higher mortality rate than females across all age categories. The mortality rate among females remained relatively steady until the age of 60 years and rapidly increased after the age of 65 years.

Life expectancy of Fabry Registry patients was calculated at 5 -year increments, beginning from the date of birth. The Fabry Registry includes patients from 39 countries, with most patients enrolled in United States (35\%) and in Europe (46\%). The United States general population was used as a reference population, where life expectancy is 74.7 years in males and 80.0 years in females. ${ }^{26}$ Life expectancy data for the European population overall are not available, but average life expectancies among 14 European countries range from 74.2 to 78.0 years in males and from 80.1 to 83.1 years in females. ${ }^{27}$ As shown in Figure 3, Fabry males had a substantially reduced life expectancy compared with the general population. At birth, the life expectancy of males was 58.2 years, compared with 74.7 years in the general population. Between birth and the age of 60 years, the average life expectancy was 17 years less in Fabry males compared with males in the general population. Over the age of 60 years, the life expectancy of Fabry males was closer to that of the general population; however, these data are based on

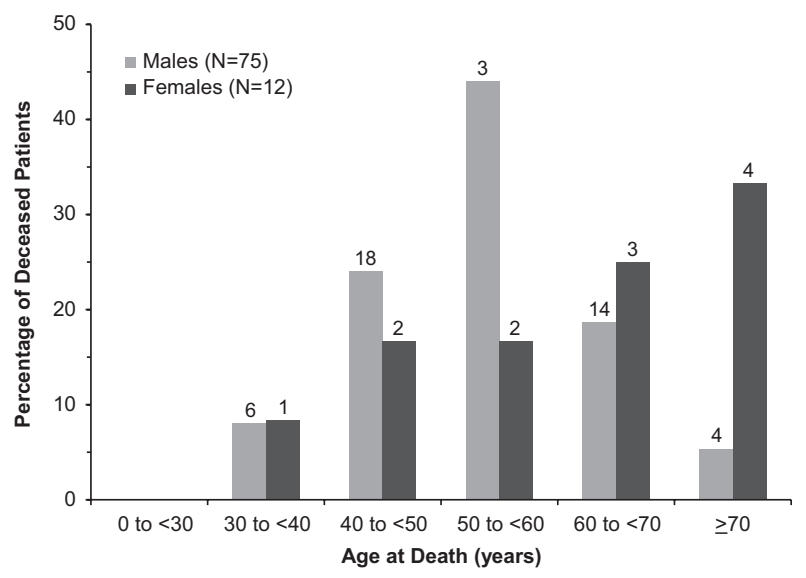

Fig. 1. Age at death for Fabry Registry patients. Percentages of patients who died during various age categories are shown. Data for males are in gray bars and data for females are in black bars, with the number of patients in each age category shown above each bar.

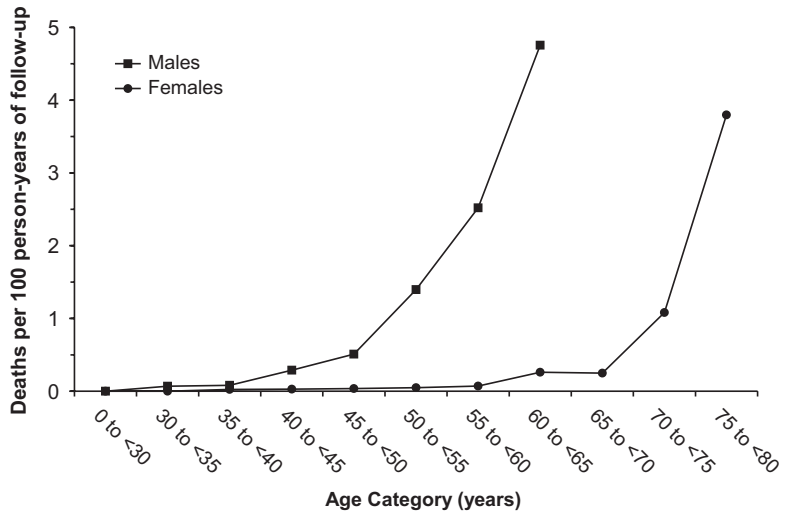

Fig. 2. Incidence of mortality in male and female Fabry Registry patients. Data are expressed as number of deaths per 100 person-years of follow-up time in various age categories. For deceased Fabry Registry patients, personyears of follow-up was defined as the number of years from birth to the date of death. For living Fabry Registry patients, person-years of follow-up was defined as the date of the last assessment. Mortality incidence was calculated by dividing the number of deaths in each age category by the total number of person-years of follow-up for each age category. Data for males are shown as squares and data for females are shown as circles.

relatively few surviving male patients. Only 93 of 1422 males in the Fabry Registry (6.5\%) were $\geq 60$ years old. The gap in life expectancy between females in the Fabry Registry and in the general population was considerably smaller. The life expectancy of Fabry females at birth was 75.4 years, compared with 80.0 years in the general population. Over all age categories, the average life expectancy was 6.4 years less in Fabry females compared with the general population. The gap widened slightly among patients over the age of 70 years, again possibly reflecting the small number of surviving Fabry females over that age: 66 of 1426 females in the Fabry Registry (4.6\%) were over the age of 70 years.

The reported cause of death was known for 56 of 75 males $(74.7 \%)$ and 10 of 12 females $(83.3 \%)$, as shown in Table 2. Cardiovascular disease was the most common cause of death among both genders; 30 of 56 males with a known cause of death $(53.6 \%)$ and 5 of 10 females with a known cause of death $(50.0 \%)$ had their deaths classified as being due to cardiovascular disease. The median age at death due to cardiovascular disease was 55.5 years in males and 66.0 years in females. Among males with a known cause of death, the second and third most common causes of death were cerebrovascular disease (7 of 56, $12.5 \%)$ and renal disease (6 of 56, 10.7\%). Among the five males who died of infection ( $8.9 \%$ of males with a known cause of death), one experienced sepsis after hemodialysis, one experienced septicemia after a renal transplant, one experienced septicemia due to a urinary tract infection, and two had pneumonia, one of whose infection was reported as being secondary to congestive heart failure. One male committed suicide at the age of 31 years. The second most common cause of death among females with a known cause of death was cancer (3 of 10, 30.0\%).

Most of the 87 deceased Fabry Registry patients had experienced a serious cardiovascular, cerebrovascular, or renal event at some time, as defined in Methods. Sixty-eight of 75 males $(90.7 \%)$ and 10 of 12 females $(83.3 \%)$ experienced 1 or more 

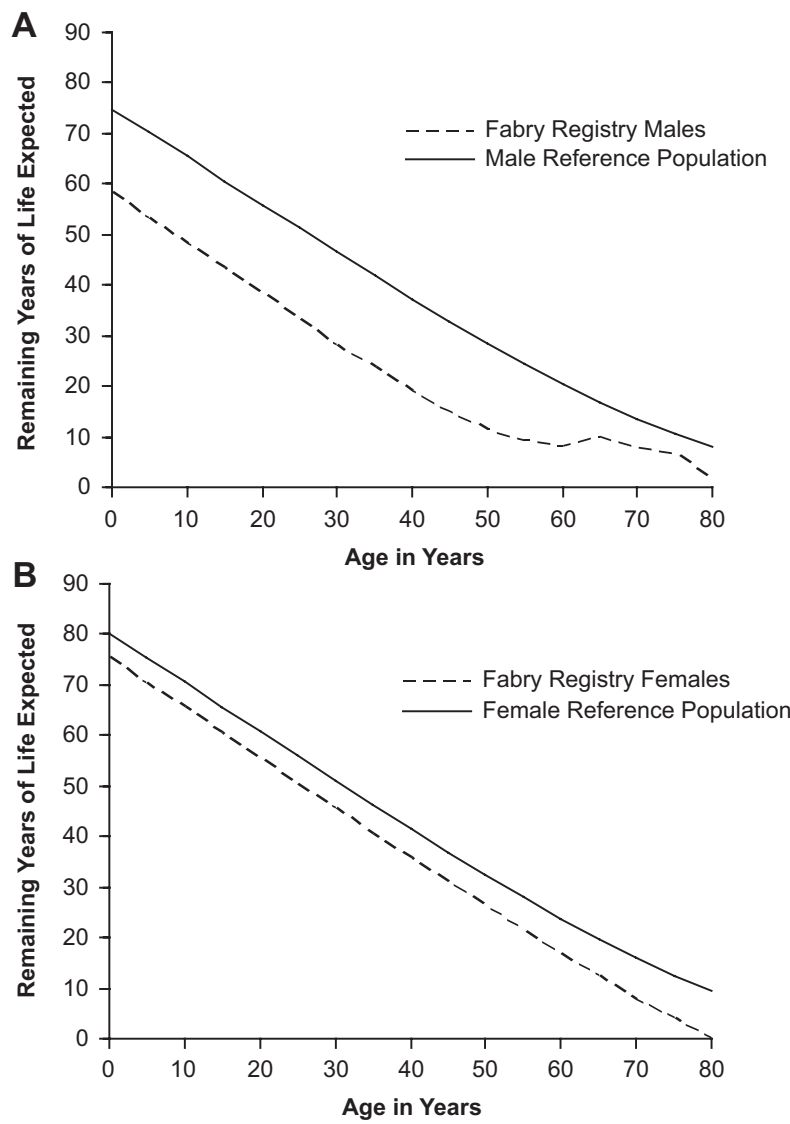

Fig. 3. Life expectancy of Fabry Registry patients. Panel A, Life expectancy of Fabry Registry males (dashed line) and males in the general US population (solid line), in years. Panel B, Life expectancy of Fabry Registry females (dashed line) and females in the general US population (solid line), in years.

clinical events before their death, as shown in Table 3. A much smaller proportion of living patients have experienced clinical events: 478 of 1347 living males (35.5\%) and 283 of 1414 living females $(20.0 \%)$. As shown in Figure 4, the most common first clinical events experienced by deceased males were renal events; 24 of $75(32.0 \%)$ received chronic dialysis and 11 of $75(14.7 \%)$ received a kidney transplant. Twenty-eight of 75 deceased males (37.3\%) experienced some type of cardiovascular event, the most common being arrhythmia (12 of 75, 16.0\%). Twelve of 75 deceased males $(16.0 \%)$ had strokes. Deceased females were more likely to have experienced cardiovascular events ( 8 of $12,66.7 \%$ ) as their first clinical event than renal events (4 of 12, 33.3\%). Arrhythmia was the most common type of first cardiovascular event among deceased females, although these data are based on relatively few patients; 4 of 12 deceased females $(33.3 \%)$ had arrhythmias.

Thus, although the most common cause of death among males was cardiovascular disease, renal events were more common as the first clinical event than were cardiovascular events. Among the 30 males whose cause of death was classified as cardiovascular, 19 $(63.3 \%)$ had also experienced a renal event at some point. Two of the five females $(40.0 \%)$ who died of cardiovascular disease had also experienced a renal event during their lives.

\section{DISCUSSION}

The Fabry Registry has collected clinical data from a large, international cohort of patients with Fabry disease, enabling the calculation of life expectancies. At birth, the life expectancy of Fabry males was 58.2 years, compared with 74.8 years in the general US population. The life expectancy of Fabry females at birth was 75.4 years, compared with 80.1 years in the general US population.

Deceased patients were much more likely to have experienced clinical events than living patients. This may be partially because the living patients were substantially younger at the time of their last follow-up visit than the deceased patients were at the time of their deaths. Deceased males had died at a median age of 54.3 years, whereas the median age at last follow-up was 37.6 years among living males. Similarly, the median age at death among females was 62.0 years, compared with a median age of 41.9 years at last follow-up visit, for living females. Thus, living patients had less time to have experienced clinical events than the older deceased patients.

Cardiovascular disease was the most common cause of death in both males and females with Fabry disease. However, renal replacement therapy (either chronic dialysis or kidney transplant) was the most common type of clinical event that was first experienced by deceased males, and most males who died of cardiovascular disease had previously received renal replacement therapy (19 of 30,63\%). Among deceased females, renal replacement therapy and arrhythmia were the most common types of first clinical event. Thus, without renal replacement therapy, many patients would have presumably died of renal failure, rather than cardiovascular disease, at much younger ages. $^{22,23}$ Renal disease in general can be both a cause and a consequence of cardiac disease. ${ }^{28,29}$ Furthermore, it has been well established that patients who receive kidney transplants or hemodialysis are at increased risk of cardiovascular disease. ${ }^{30,31}$ Consequently, the cardiovascular and renal manifestations of Fabry disease may be at least partially interrelated. The impact and pathophysiology of Fabry disease is well known and has been the focus of many studies. ${ }^{3-13,17}$ However, little is known about the progressive effect of Fabry disease on the cardiovascular system. It has been established that Fabry disease leads to GL-3 accumulation in the vascular endothelium of the heart, as well as within cardiomyocytes. ${ }^{15,32}$ It is not known whether the deaths due to cardiovascular disease in this cohort were caused primarily by cardiovascular complications of Fabry disease itself or due to complications of underlying renal disease. Based on the involvement of the vascular endothelium and the myocardium, Fabry disease may lead to cardiovascular death via multiple pathways

Five males were reported to have died of infection. There is no evidence that patients with Fabry disease are particularly susceptible to infection. It is likely that some of these infections arose as complications of the various medical procedures that patients with advanced Fabry disease require. One patient died of sepsis after hemodialysis and a second died of septicemia after a renal transplant. In addition, cardiovascular disease is a known risk factor for pneumonia, which was reported as the cause of death in 2 males.

The patients who died were diagnosed with Fabry disease at a relatively late age: median 40 years in males and 55 years in females. Accordingly, the disease had progressed substantially before these patients were diagnosed, which likely contributed to their early deaths. Fortunately, patients today tend to be diagnosed with Fabry disease at a much earlier age; in 2007, the median age at diagnosis among the overall population of Fabry 
Table 2 Cause of death among Fabry Registry patients

\begin{tabular}{|c|c|c|c|c|}
\hline & \multicolumn{2}{|c|}{ Deceased males $(N=75)$} & \multicolumn{2}{|c|}{ Deceased females $(N=12)$} \\
\hline & No. deaths, $n(\%)$ & Median age at death (yr) & No. deaths, $n(\%)$ & Median age at death (yr) \\
\hline \multicolumn{5}{|l|}{ Cause of death } \\
\hline Cardiovascular & $30(40.0)$ & 55.5 & $5(41.7)$ & 66.0 \\
\hline $\begin{array}{l}\text { Unknown or not } \\
\text { reported }\end{array}$ & $19(25.3)$ & 53.8 & $2(16.7)$ & 56.0 \\
\hline Cerebrovascular & $7(9.3)$ & 49.3 & $1(8.3)$ & 56.7 \\
\hline Renal & $6(8.0)$ & 55.5 & $1(8.3)$ & 74.3 \\
\hline Infection & $5(6.7)$ & 42.2 & 0 & - \\
\hline Gastrointestinal & $3(4.0)$ & 44.9 & 0 & - \\
\hline Cancer & $2(2.7)$ & 61.1 & $3(25.0)$ & 63.3 \\
\hline Respiratory & $1(1.3)$ & 71.5 & 0 & - \\
\hline Suicide & $1(1.3)$ & 31.2 & 0 & - \\
\hline Other $^{a}$ & $1(1.3)$ & 47.9 & 0 & - \\
\hline
\end{tabular}

Percentages were calculated based on the total number of deceased patients, including those for whom a cause of death was unknown or not reported. Two males and one female Fabry Registry patients who were deceased but who did not have a date of death reported were excluded from the dataset.

${ }^{a}$ One male died after suffering multiorgan failure during hip surgery (sepsis, hepatic insufficiency, and cardiorespiratory failure).

Table 3 Age at first clinical event in deceased and living Fabry Registry patients

\begin{tabular}{|c|c|c|c|c|}
\hline \multirow[b]{2}{*}{ Parameter } & \multicolumn{2}{|c|}{ Males } & \multicolumn{2}{|c|}{ Females } \\
\hline & $\begin{array}{l}\text { Deceased } \\
(N=75)\end{array}$ & $\begin{array}{c}\text { Alive } \\
(N=1347)\end{array}$ & $\begin{array}{l}\text { Deceased } \\
(N=12)\end{array}$ & $\begin{array}{c}\text { Alive } \\
(N=1414)\end{array}$ \\
\hline Ever experienced any clinical event, $n(\%)$ & $68(90.7)$ & $478(35.5)$ & $10(83.3)$ & $283(20.0)$ \\
\hline \multicolumn{5}{|l|}{ Age at first clinical event (yr) } \\
\hline Mean (SD) & $43.3(10.57)$ & $37.8(11.55)$ & $52.3(13.03)$ & $46.7(13.49)$ \\
\hline Median (range) & $43.5(16.8-76.8)$ & $38.3(5.30-80.4)$ & $49.9(31.2-74.8)$ & $47.2(17.0-78.2)$ \\
\hline Ever experienced renal event, $n(\%)$ & $44(64.7)$ & $216(45.1)$ & $4(40.0)$ & $30(10.6)$ \\
\hline \multicolumn{5}{|l|}{ Age at first renal event (yr) } \\
\hline Mean (SD) & $43.3(10.25)$ & $39.4(10.03)$ & $43.0(8.29)$ & $40.8(12.21)$ \\
\hline Median (range) & $42.3(16.8-79.1)$ & $39.9(14.8-74.0)$ & $45.0(31.2-50.6)$ & $39.5(17.0-77.8)$ \\
\hline Ever experienced cerebrovascular event, $n(\%)$ & $28(41.2)$ & $110(22.3)$ & $3(30.0)$ & $69(24.4)$ \\
\hline \multicolumn{5}{|l|}{ Age at first cerebrovascular event (yr) } \\
\hline Mean (SD) & $47.0(11.13)$ & $40.2(11.88)$ & $60.1(6.74)$ & $47.1(14.5)$ \\
\hline Median (range) & $48.3(25.8-80.1)$ & $39.5(13.8-73.6)$ & $56.7(55.8-67.9)$ & $46.7(19.8-74.8)$ \\
\hline Ever experienced cardiac event, $n(\%)$ & $43(63.2)$ & $334(69.9)$ & $8(80.0)$ & $231(81.6)$ \\
\hline \multicolumn{5}{|l|}{ Age at first cardiac event (yr) } \\
\hline Mean (SD) & $49.7(9.26)$ & $40.2(12.38)$ & $55.5(11.46)$ & $48.6(13.03)$ \\
\hline Median (range) & $50.6(25.6-76.8)$ & $41.7(5.3-80.4)$ & $50.3(47.5-74.8)$ & $49.8(17.3-78.2)$ \\
\hline
\end{tabular}

Percentages of patients who experienced renal, cerebrovascular, or cardiac events were calculated based on the number of patients in that category who ever experienced any clinical event.

Registry patients was 24 years in males and 31 years in females. ${ }^{10}$ As cardiovascular disease was the most common cause of death, cardiac function should be closely monitored in patients with Fabry disease. All patients should be routinely monitored by standard and 24-hour electrocardiogram and echocar- diogram, regardless of gender or whether they exhibit signs of cardiac disease. ${ }^{33}$ In addition, cardiologists should consider the possibility of Fabry disease in patients with hypertrophic cardiomyopathy (HCM). A study of 153 males with HCM identified 6 patients (4\%) who had undiagnosed Fabry disease. ${ }^{34} \mathrm{~A}$ 


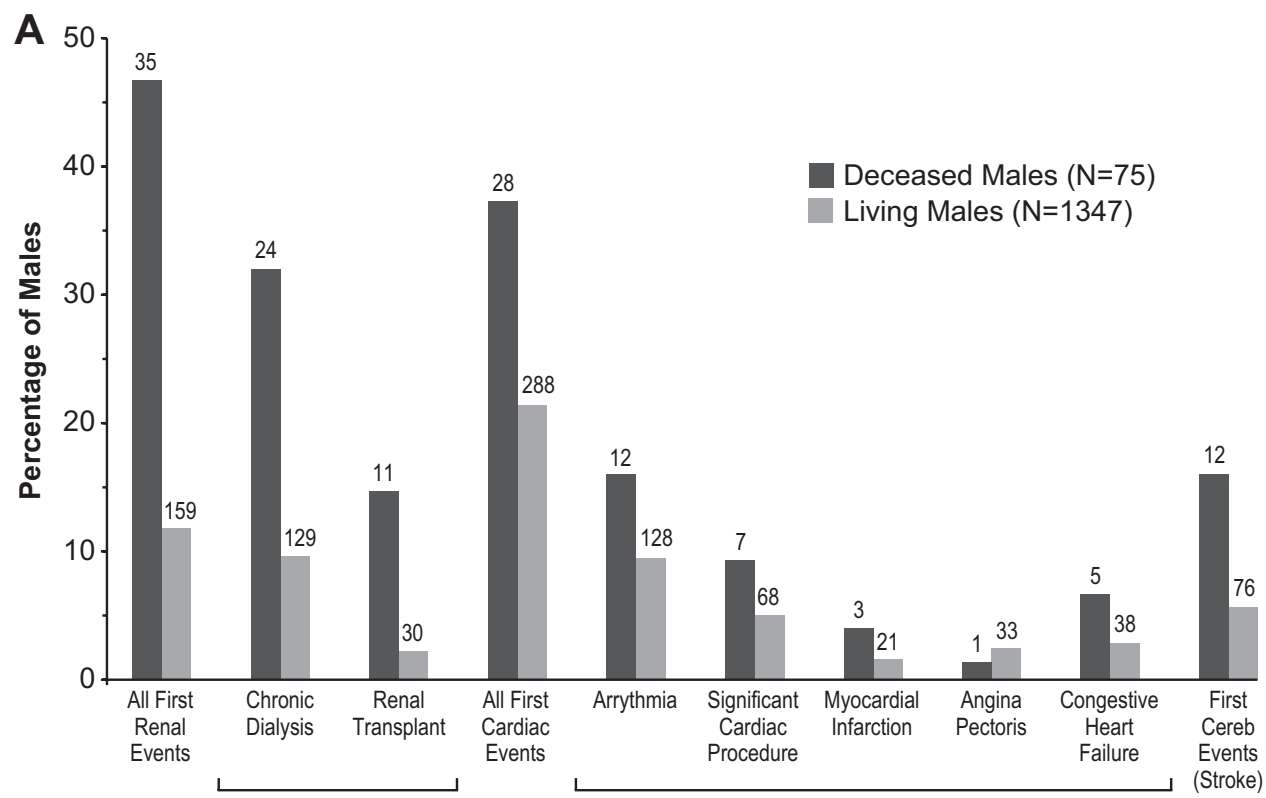

Type of First Clinical Event

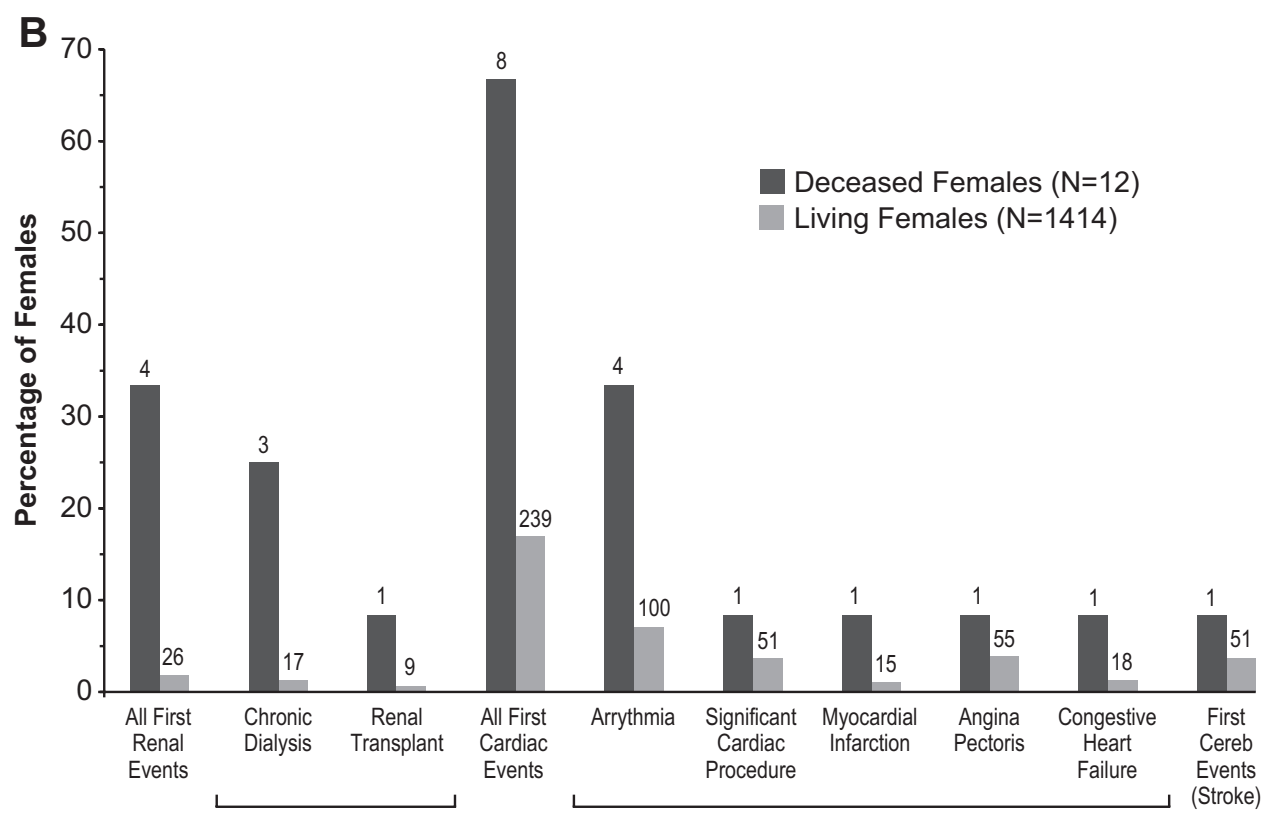

Type of First Clinical Event

Fig. 4. Type of significant clinical events first experienced by Fabry Registry patients. Panel A, Data are expressed as the percentage of all deceased or living Fabry Registry males who experienced the indicated events as their first clinical event. Panel B, Data are expressed as the percentage of all deceased or living Fabry Registry females who experienced the indicated events as their first clinical event. Data for deceased patients are shown in black bars, and data for living patients are shown in gray bars, with the number of patients in each age category shown above each bar. The percentages of all patients with renal events and all patients with cardiac events are shown along with the percentages of patients with specific types of renal and cardiac events (indicated in brackets). Note that six deceased males and two deceased females are included in multiple categories, because they experienced more than one type of first event on the same date. Cereb, cerebrovascular.

second screening study of 508 patients with HCM, including 328 males and 180 females, reported that $1 \%$ of patients had undiagnosed Fabry disease. ${ }^{35}$

It is important to note that 61 of the 75 deceased males $(81.3 \%)$ and 5 of the 12 deceased females $(41.7 \%)$ in the Fabry
Registry were known to have received ERT at some point. These patients were untreated for the majority of their lives, as the median duration of ERT was 12 months in males (range, 0.1-7.3 years) and 4 months in females (range, $0.1-2.8$ years). As noted earlier, the deceased patients were diagnosed at a 
relatively late age and were therefore likely to have been in the advanced stages of Fabry disease at the time of diagnosis, as well as at the time they began receiving ERT.

A recent report by Mehta et al. ${ }^{24}$ evaluated the cause of death in patients enrolled in the Fabry Outcome Survey (FOS). Similar to the Fabry Registry findings, Mehta et al. ${ }^{24}$ reported that the most common cause of death was cardiovascular disease $(36 \%)$, followed by infection (15\%), among the 42 patients whose deaths were reported between 2001 and 2007. The mean ages at death are generally similar in the two studies: 51.8 years in FOS males versus 53.7 years in Fabry Registry males and 64.4 years in FOS females versus 60.9 years in Fabry Registry females. Neither life expectancy nor the age at diagnosis of deceased patients was reported by Mehta et al. The findings from both studies suggest that recent improvements in treating renal disease have led to a decrease in Fabry patient deaths due to renal failure; more patients currently die of cardiovascular disease.

The findings from these analyses are limited by the observational nature of data reported to registries. For example, although a standardized definition of clinical events is provided in the Fabry Registry case report form, an independent committee did not adjudicate all events. In addition, patients may be lost to follow-up and/or the occurrence or timing of clinical events or death may not be captured.

Earlier diagnosis, guidelines for treating children, and the broader availability of ERT will enable patients to be treated earlier, well before reaching advanced stages of cardiovascular or renal disease. Previous studies have shown that ERT most effectively slows the progression of renal decline ${ }^{18,19,36}$ and cardiomyopathy ${ }^{20,21}$ when patients are treated early during the course of the disease. Accordingly, earlier diagnosis and initiation of treatment would be expected to extend survival. The findings of these analyses highlight the burden of Fabry disease, which frequently leads to multiple serious clinical events and is associated with reduced lifespan. Physicians caring for patients with Fabry disease should continue to vigilantly evaluate and identify disease progression and end-organ involvement, to institute treatments aimed at improving patient outcomes.

\section{ACKNOWLEDGMENTS}

The Fabry Registry is sponsored by Genzyme Corporation. The authors thank the many patients that have agreed to participate in the Fabry Registry as well as the physicians and research coordinators that have entered clinical data on these patients. The authors also acknowledge our colleagues at Genzyme Corporation (Cambridge, MA), Dana BeitnerJohnson, $\mathrm{PhD}$, and Fanny O'Brien, $\mathrm{PhD}$, for assistance with manuscript writing and statistical analyses.

\section{REFERENCES}

1. Desnick RJ, Ioannou YA, Eng CM. $\alpha$-Galactosidase a deficiency: Fabry disease. In: Scriver CR, Beaudet AL, Sly WS, ValleD, editors. The metabolic and molecular bases of inherited disease. 8th ed. New York: McGraw Hill, 2001:3733-3774.

2. Meikle PJ, Hopwood JJ, Clague AE, et al. Prevalance of lysosomal storage disorders. JAMA 1999;281:249-254.

3. Ries M, Ramaswami U, Parini R, et al. The early clinical phenotype of Fabry disease: a study on 35 European children and adolescents. Eur J Pediatr 2003; $162: 767-772$

4. Ries M, Gupta S, Moore DF, et al. Pediatric Fabry disease. Pediatrics 2005; 115:e344-e355.

5. Hopkin RJ, Bissler J, Banikazemi M, et al. Characterization of Fabry disease in 352 pediatric patients in the Fabry Registry. Pediatr Res 2008;64:550555

6. MacDermot KD, Holmes A, Miners AH. Anderson-Fabry disease: clinical manifestations and impact of disease in a cohort of 98 hemizygous males. $J$ Med Genet 2001;38:750-760.

7. MacDermot KD, Holmes A, Miners AH. Anderson-Fabry disease: clinical manifestations and impact of disease in a cohort of 60 obligate carrier females. J Med Genet 2001;38:769-775.

8. Branton MH, Schiffmann R, Sabnis SG, et al. Natural history of Fabry renal disease: influence of alpha-galactosidase A activity and genetic mutations on clinical course. Medicine (Baltimore) 2002;81:122-138.

9. Mehta A, Ricci R, Widmer U, et al. Fabry disease defined: baseline clinical manifestations of 366 patients in the Fabry Outcome Survey. Eur J Clin Invest 2004;34:236-242.

10. Wilcox WR, Oliveira JP, Hopkin RJ, et al. Females with Fabry disease frequently have major organ involvement: lessons from the Fabry Registry. Mol Genet Metab 2008;93:112-128.

11. Sims K, Politei J, Banikazemi M, Lee P. Stroke in Fabry disease frequently occurs before diagnosis and in the absence of other clinical events: natural history data from the Fabry Registry. Stroke 2009;40:788-794

12. Wang RY, Lelis A, Mirocha J, Wilcox WR. Heterozygous Fabry women are not just "carriers," but have a significant burden of disease and impaired quality of life. Genet Med 2007;9:34-45.

13. Vedder AC, Linthorst GE, van Breemen MJ, et al. The Dutch Fabry cohort: diversity of clinical manifestations and Gb3 levels. J Inherit Metab Dis 2007;30:68-78

14. Eng CM, Banikazemi M, Gordon RE, et al. A phase $1 / 2$ clinical trial of enzyme replacement in Fabry disease: pharmacokinetic, substrate clearance, and safety studies. Am J Hum Genet 2001;68:711-722.

15. Eng CM, Guffon N, Wilcox WR, et al; International Collaborative Fabry Disease Study Group. Safety and efficacy of recombinant human alphagalactosidase A-replacement therapy in Fabry's disease. $N$ Engl $\mathrm{J} \mathrm{Med}$ 2001;345:9-16.

16. Schiffmann R, Kopp JB, Austin HA III, et al. Enzyme replacement therapy in Fabry disease: a randomized controlled trial. JAMA 2001;285:2743-2749.

17. Wilcox WR, Banikazemi M, Guffon N, et al. Long-term safety and efficacy of enzyme replacement therapy for Fabry disease. Am J Hum Genet 2004; 75:65-74

18. Germain DP, Waldek S, Banikazemi M, et al. Sustained, long-term renal stabilization after 54 months of agalsidase beta therapy in patients with Fabry disease. J Am Soc Nephrol 2007;18:1547-1557.

19. West M, Nicholls K, Mehta A, et al. Agalsidase alfa and kidney dysfunction in Fabry disease. J Am Soc Nephrol 2009;20:1132-1139.

20. Hughes DA, Elliott PM, Shah J, et al. Effects of enzyme replacement therapy on the cardiomyopathy of Anderson-Fabry disease: a randomised, doubleblind, placebo controlled clinical trial of agalsidase alfa. Heart 2008;94: $153-158$.

21. Weidemann F, Niemann M, Breunig F, et al. Long-term effects of enzyme replacement therapy on Fabry cardiomyopathy: evidence for a better outcome with early treatment. Circulation 2009;119:524-529.

22. Wise D, Wallace HJ, Jellinek EH. Angiokeratoma corporis diffusum. Q J Med 1962;31:177-206.

23. Colombi A, Kostyal A, Bracher R, et al. Angiokeratoma corporis diffusumFabry's disease. Helv Med Acta 1967;34:67-83.

24. Mehta A, Clark JTR, Giugliani R, et al; FOS Investigators. Natural course of Fabry diseases: changing pattern of causes of death in FOS - the Fabry Outcome Survey. J Med Genet 2009;46:548-552.

25. Selvin S. Statistical analysis of epidemiologic data. New York: Oxford University Press, 1991:241-244.

26. Hoyert DL, Heron MP, Murphy SL, Kung HC. Deaths: final data for 2003. Natl Vital Stat Rep 2006;54:1-120.

27. Jagger C. EHEMU team; Healthy life expectancy in the EU15. Available at: http://www.ehemu.eu/index.php?option=reports. Accessed April 22, 2009.

28. Go AS, Chertow GM, Fan D, McCulloch CE, Hsu CY. Chronic kidney disease and the risks of death, cardiovascular events, and hospitalization. $N$ Engl J Med 2004;351:1296-1305.

29. Elsayed EF, Tighiouart H, Griffith, et al. Cardiovascular disease and subsequent kidney disease. Arch Intern Med 2007;167:1130-1136.

30. Dimény EM. Cardiovascular disease after renal transplantation. Kidney Int Suppl 2002;80:78-84.

31. Wanner C, Zimmermann J, Schwedler S, Metzger T. Inflammation and cardiovascular risk in dialysis patients. Kidney Int Suppl 2002;80:99-102.

32. Chimenti C, Hamdani N, Boontje NM, et al. Myofilament degradation and dysfunction of human cardiomyocytes in Fabry disease. Am J Pathol 2008; 172:1482-1490.

33. Eng CM, Germain DP, Banikazemi M, et al. Fabry disease: guidelines for the evaluation and management of multi-organ system involvement. Genet Med 2006;8:539-548.

34. Sachdev B, Takenaka T, Teraguchi H, et al. Prevalence of AndersonFabry disease in male patients with late onset hypertrophic cardiomyopathy. Circulation 2002;105:1407-1411.

35. Monserrat L, Gimeno-Blanes JR, Marín F, et al. Prevalence of Fabry disease in a cohort of 508 unrelated patients with hypertrophic cardiomyopathy. J Am Coll Cardiol 2007;50:2399-2403.

36. Banikazemi M, Bultas J, Waldek S, et al; Fabry Disease Clinical Trial Study Group. Agalsidase-beta therapy for advanced Fabry disease. Ann Intern Med 2007; 146:77-86. 